PROCEEDINGS OF THE

AMERICAN MATHEMATICAL SOCIETY

Volume 134, Number 8, Pages 2329-2334

S 0002-9939(06)08395-X

Article electronically published on February 6, 2006

\title{
INTERPOLATION THEOREM ON LORENTZ SPACES OVER WEIGHTED MEASURE SPACES
}

\author{
SHINYA MORITOH, MIYUKI NIWA, AND TAKUYA SOBUKAWA
}

(Communicated by Christopher D. Sogge)

\begin{abstract}
In 1997 Ferreyra proved that it is impossible to extend the SteinWeiss theorem in the context of Lorentz spaces. In this paper we obtain an interpolation theorem on Lorentz spaces over weighted measure spaces.
\end{abstract}

\section{INTRODUCTION}

The aim of the paper is to prove an interpolation theorem on Lorentz spaces over weighted measure spaces.

In 1966, Calderón 2 and Hunt 4 proved an interpolation theorem on Lorentz spaces $L_{p, q}$. On the other hand, in 1958, Stein and Weiss 7 proved an interpolation theorem on $L_{p}$ spaces which allows one to change measures simultaneously with changing exponents. Thus it is natural to seek an interpolation theorem on $L_{p, q}$ spaces which allows one to change measures simultaneously with changing exponents. However, in 1997, Ferreyra [3] gave a negative result for such an interpolation.

In [5, the following result was obtained; notation is given in section 2 .

Let $i=0,1$. Suppose that $0<p_{0}<p_{1} \leq \infty, 0<r_{i} \leq \infty$ with $r_{0} \neq$ $r_{1}, 0<q_{i} \leq \infty, 0<s_{i} \leq \infty$ and $\mathbb{D}=(-\alpha,-\alpha) \in \mathbb{R}^{2}$ with $\alpha>1$. Put $1 / p=$ $(1-\theta) / p_{0}+\theta / p_{1}, 1 / r=(1-\theta) / r_{0}+\theta / r_{1}$ for $0<\theta<1$. Let $v_{i}, w_{i}$ be nonnegative measurable functions. If $T$ is a quasi-linear operator bounded from $L_{p_{i}, q_{i} ; v_{i}}$ to $L_{r_{i}, s_{i} ; w_{i}}$, then there exists a constant $C$ such that $\|T f\|_{r, s ; \mathbb{D} ; w} \leq C\|f\|_{p, q ; v}$ for every linear combination $f$ of two characteristic functions with disjoint supports, where $q \leq s$ and $v^{1 / p}=v_{0}^{(1-\theta) / p_{0}} v_{1}^{\theta / p_{1}}, w^{1 / r}=w_{0}^{(1-\theta) / r_{0}} w_{1}^{\theta / r_{1}}$.

In this paper, we prove an interpolation theorem of the above type. Under the hypothesis that $T$ is nonnegative, and bounded from $L_{p_{i}, q_{i} ; v}$ to $L_{r_{i}, s_{i} ; w_{i}}$, we obtain the inequality $\|T f\|_{r, \infty ; w} \leq C\|f\|_{p, 1 ; v}$. Note that the domain space is a Lorentz space $L_{p, 1 ; v}$ with fixed weight $v$. This conclusion is weak in the sense that the target $L_{r, \infty}$ is large and the domain $L_{p, 1}$ is small in the framework of $L_{p, q}$ classes.

The plan of the paper is as follows. In preliminary section 2 we give definitions and lemmas. In section 3 we state our main theorem and give its proof.

Received by the editors January 10, 2003 and, in revised form, March 8, 2005.

2000 Mathematics Subject Classification. Primary 46B70; Secondary 46E30.

Key words and phrases. Weighted Lorentz spaces, weighted interpolation.

(C)2006 American Mathematical Society Reverts to public domain 28 years from publication 


\section{Preliminaries}

Let $(M, \mathfrak{M}, \mu)$ be a resonant measure space and let $f$ be a complex-valued or real-valued measurable function defined on $M$.

Definition 2.1. Let $w$ be a nonnegative measurable function on $M$. Then the distribution function of $f$ with respect to $w$ is defined as follows:

$$
\lambda_{f, w}(y)=w(\{x \in M:|f(x)|>y\}), \quad y>0,
$$

where $w(A)=\int_{A} w d \mu$ for every $A \in \mathfrak{M}$. Then the nonincreasing rearrangement of $f$ with respect to $w$ is defined as follows:

$$
f_{w}^{*}(t)=\inf \left\{y: \lambda_{f, w}(y) \leq t\right\}, \quad t>0 .
$$

The average function of $f$ with respect to $w$ is defined as follows:

$$
f_{w}^{* *}(t)=\frac{1}{t} \int_{0}^{t} f_{w}^{*}(y) d y, \quad t>0 .
$$

Definition 2.2. Let $w$ be a nonnegative measurable function on $M$. Then the weighted Lorentz space $L_{p, q ; w}$ is defined as the set of all measurable functions $f$ on $M$ such that $\|f\|_{p, q ; w}<\infty$, where

$$
\|f\|_{p, q ; w}= \begin{cases}\left(\frac{q}{p} \int_{0}^{\infty}\left[t^{1 / p} f_{w}^{*}(t)\right]^{q} \frac{d t}{t}\right)^{1 / q}, & 1 \leq p<\infty, 0<q<\infty, \\ \sup _{t>0} t^{1 / p} f_{w}^{*}(t), & 1 \leq p \leq \infty, q=\infty .\end{cases}
$$

In general, however, $\|\cdot\|_{p, q ; w}$ is not a norm since the Minkowski inequality may fail. But by replacing $f_{w}^{*}$ with $f_{w}^{* *}$ in the above definition of $\|f\|_{p, q ; w}$, we obtain a norm $\|\cdot\|_{(p, q) ; w}$ for all $q \geq 1$ :

$$
\|f\|_{(p, q) ; w}= \begin{cases}\left(\frac{q}{p} \int_{0}^{\infty}\left[t^{1 / p} f_{w}^{* *}(t)\right]^{q} \frac{d t}{t}\right)^{1 / q}, & 1<p<\infty, 0<q<\infty, \\ \sup _{t>0} t^{1 / p} f_{w}^{* *}(t), & 1<p \leq \infty, q=\infty .\end{cases}
$$

We state the following from Bennett and Sharpley [1].

Lemma 2.3. If $1<p \leq \infty$ and $1 \leq q \leq \infty$, then

$$
\|f\|_{p, q ; w} \leq\|f\|_{(p, q) ; w} \leq \frac{p}{p-1}\|f\|_{p, q ; w} .
$$

Proof. The first inequality is an immediate consequence of Definition 2.2 and the fact that $f_{w}^{*} \leq f_{w}^{* *}$. The second follows from the Hardy inequality.

As mentioned in the introduction, we include the definition of Lorentz-Zygmund spaces with norm $\|\cdot\|_{p, q ; \mathbb{D} ; w}$. We note that the case where $\mathbb{D}=(0,0)$ corresponds to the weighted Lorentz spaces given in Definition 2.2.

Definition 2.4. Let $w$ be a nonnegative measurable function on $M$. Then the weighted Lorentz-Zygmund space $L_{p, q ; w}(\log L)_{\mathbb{D}}, \mathbb{D}=(\alpha, \beta) \in \mathbb{R}^{2}$, is defined as the set of all measurable functions $f$ on $M$ such that $\|f\|_{p, q ; \mathbb{D} ; w}<\infty$, where

$$
\|f\|_{p, q ; \mathbb{D} ; w}= \begin{cases}\left(\frac{q}{p} \int_{0}^{\infty}\left[t^{1 / p} f_{w}^{*}(t)\right]^{q}(\log t)^{\mathbb{D}} \frac{d t}{t}\right)^{1 / q}, & 0<p, q<\infty, \\ \sup _{t>0} t^{1 / p} f_{w}^{*}(t)(\log t)^{\mathbb{D}}, & 0<p \leq \infty, q=\infty .\end{cases}
$$


Here,

$$
(\log t)^{\mathbb{D}}= \begin{cases}(1-\log t)^{\alpha}, & 0<t<1, \\ (1+\log t)^{\beta}, & 1 \leq t<\infty .\end{cases}
$$

Let $(N, \mathfrak{N}, \nu)$ be another resonant measure space. Let $T$ be an operator from a class of measurable functions on $M$ to that on $N$.

Definition 2.5. An operator $T$ is called quasi-linear if there is a constant $K \geq 1$ such that

$$
|T(f+g)| \leq K(|T f|+|T g|), \quad|T(\lambda f)|=|\lambda| \cdot|T f|
$$

hold $\nu$-a.e. on $N$ for all $f$ and $g$ in the domain of $T$ and for all scalars $\lambda$. If we can take $K=1, T$ is called sublinear. $T$ is called nonnegative when $T f \geq 0, \nu$-a.e. for all $f$ in the domain of $T$.

We recall the following result from Bennett and Sharpley [1, Lemma 5.1, p. 231]. This is necessary for the limiting argument given in the proof of Theorem 3.1 in the next section 3 .

Lemma 2.6. Let $X$ and $Y$ be rearrangement-invariant Banach function spaces over resonant measure spaces $(M, \mu)$ and $(N, \nu)$, respectively. Let $T$ be a nonnegative sublinear operator defined on a dense linear subspace $D$ of $X$ and taking values in $Y$. If

$$
\|T f\|_{Y} \leq C\|f\|_{X}
$$

for all $f \in D$, then $T$ has a unique extension to a nonnegative sublinear operator from $X$ to $Y$, for which (2.1) holds for all $f \in X$.

Finally, we prove the following.

Lemma 2.7. There are constants $C_{1}$ and $C_{2}$ such that

$$
C_{1} \sum_{k=-\infty}^{\infty} 2^{k / p} f_{w}^{*}\left(2^{k}\right) \leq\|f\|_{p, 1 ; w} \leq C_{2} \sum_{k=-\infty}^{\infty} 2^{k / p} f_{w}^{*}\left(2^{k}\right) .
$$

Proof. Let $t \in\left[2^{k}, 2^{k+1}\right]$. Since $f_{w}^{*}$ is nonincreasing, $f_{w}^{*}\left(2^{k+1}\right) \leq f_{w}^{*}(t) \leq f_{w}^{*}\left(2^{k}\right)$.

On the one hand, we obtain

$$
\begin{aligned}
\|f\|_{p, 1 ; w} & =\frac{1}{p} \int_{0}^{\infty} t^{1 / p} f_{w}^{*}(t) \frac{d t}{t} \\
& =\frac{1}{p} \sum_{k=-\infty}^{\infty} \int_{2^{k}}^{2^{k+1}} t^{1 / p} f_{w}^{*}(t) \frac{d t}{t} \\
& \leq \frac{1}{p} \sum_{k=-\infty}^{\infty} \int_{2^{k}}^{2^{k+1}} t^{1 / p} f_{w}^{*}\left(2^{k}\right) \frac{d t}{t} \\
& =\sum_{k=-\infty}^{\infty} f_{w}^{*}\left(2^{k}\right)\left(2^{(k+1) / p}-2^{k / p}\right) \\
& =\left(2^{1 / p}-1\right) \sum_{k=-\infty}^{\infty} 2^{k / p} f_{w}^{*}\left(2^{k}\right) .
\end{aligned}
$$


On the other hand, we obtain

$$
\begin{aligned}
\|f\|_{p, 1 ; w} & \geq \frac{1}{p} \sum_{k=-\infty}^{\infty} \int_{2^{k}}^{2^{k+1}} t^{1 / p} f_{w}^{*}\left(2^{k+1}\right) \frac{d t}{t} \\
& =\sum_{k=-\infty}^{\infty} f_{w}^{*}\left(2^{k+1}\right)\left(2^{(k+1) / p}-2^{k / p}\right) \\
& =\left(1-2^{-1 / p}\right) \sum_{k=-\infty}^{\infty} 2^{(k+1) / p} f_{w}^{*}\left(2^{k+1}\right) .
\end{aligned}
$$

\section{MAIN THEOREM}

We can now prove the following.

Theorem 3.1. Let $i=0,1$. Suppose that $1 \leq p_{0}<p_{1} \leq \infty, 1 \leq r_{i} \leq \infty$ with $r_{0} \neq r_{1}, 0<q_{i}, s_{i} \leq \infty$. Put $1 / p=(1-\theta) / p_{0}+\theta / p_{1}, 1 / r=(1-\theta) / r_{0}+\theta / r_{1}$ for $0<\theta<1$. Let $v, w_{i}$ be nonnegative measurable functions. If $T$ is a nonnegative sublinear operator from $L_{p_{i}, q_{i} ; v}$ to $L_{r_{i}, s_{i} ; w_{i}}$, then there exists a constant $C$ such that

$$
\|T f\|_{r, \infty ; w} \leq C\|f\|_{p, 1 ; v},
$$

where $w^{1 / r}=w_{0}^{(1-\theta) / r_{0}} w_{1}^{\theta / r_{1}}$.

Remark 3.2. We do not know if this theorem is true in the case where $T$ is not assumed to be nonnegative.

Proof of Theorem 3.1. In our proof, the letter $C$ will denote a constant not necessarily the same at each occurrence. Let $f$ be a measurable function on $M$. We consider pairwise disjoint measurable subsets of $M$,

$$
E_{n}=\left\{x \in M: f_{v}^{*}\left(2^{n+1}\right)<|f(x)| \leq f_{v}^{*}\left(2^{n}\right)\right\}, \quad n=0, \pm 1, \pm 2, \ldots .
$$

We may assume that $|f(x)|<\infty$, $\mu$-a.e. $x \in M$. If $|f(x)| \equiv \infty$ on some set of positive measure, then we have $f_{v}^{*}(t) \equiv \infty$ near $t=0$, which implies that $\|f\|_{p, 1 ; v}=\infty$. Moreover, we may also assume that $f_{v}^{*}(t)$ tends to 0 as $t \rightarrow \infty$. If $f_{v}^{*}(t)$ does not tend to 0 , then $\|f\|_{p, 1 ; v}=\infty$.

Let $f_{n}$ be defined by

$$
f_{n}(x)= \begin{cases}f(x), & x \in E_{n}, \\ 0, & \text { otherwise }\end{cases}
$$

Then we consider the following three cases according to the value of $|f(x)|$. When $|f(x)|=0, x$ does not belong to any $E_{n}$, so $f_{n}(x)=0$ for every $n$. Next, when $0<|f(x)|<\infty, x$ belongs to some $E_{n}$ because we have assumed that $f_{v}^{*}(t)$ tends to 0 as $t \rightarrow \infty$. In this case, $f_{n}(x)=f(x)$ and $f_{m}(x)=0$ for $m \neq n$. Finally, when $|f(x)|=\infty, x$ does not belong to any $E_{n}$ because we have assumed that $|f(x)|<\infty, \mu$-a.e. $x \in M$. In this final case, $f_{n}(x)=0$ for every $n$. Therefore, we may describe the function $f$ as the following sum of the functions $f_{n}$ (see [6]):

$$
f(x)=\sum_{n=-\infty}^{\infty} f_{n}(x), \quad \mu \text {-a.e. } x \in M .
$$


We remark that if $E_{n}=\emptyset$, then $f_{n}(x) \equiv 0$ on $M$. Another important remark is that we have $v\left(E_{n}\right) \leq 2 \cdot 2^{n}$ because of the construction of the sets $E_{n}$, which is used in the estimate (3.6) below. Because of Lemma 2.6. we may assume that all but finitely many $f_{n}$ 's in (3.1) are identically zero. The set of such functions $f$ is a dense linear subspace of $L_{p, 1 ; v}, 1<p<\infty$, and we can apply a limiting argument.

By using the sublinearity of $T$ (Definition 2.5), the Minkowski inequality (Definition 2.2) and Lemma 2.3. we have

$$
\|T f\|_{(r, \infty) ; w} \leq \sum_{n=-\infty}^{\infty}\left\|T f_{n}\right\|_{(r, \infty) ; w} \leq \frac{r}{(r-1)} \sum_{n=-\infty}^{\infty}\left\|T f_{n}\right\|_{r, \infty ; w} .
$$

(We note again that the summations in the inequalities in (3.2) have only finitely many nonzero terms.)

We now estimate the quantity $\left\|T f_{n}\right\|_{r, \infty ; w}$. Because $T$ is assumed to be a sublinear operator from $L_{p_{i}, q_{i} ; v}$ to $L_{r_{i}, s_{i} ; w_{i}}$,

$$
t^{1 / r_{i}}\left(T f_{n}\right)_{w_{i}}^{*}(t) \leq C\left\|f_{n}\right\|_{p_{i}, q_{i} ; v}, \quad i=0,1
$$

By the change of variables $t=\lambda_{T f_{n}, w_{i}}(s)$, we have

$$
s\left(\lambda_{T f_{n}, w_{i}}(s)\right)^{1 / r_{i}} \leq C\left\|f_{n}\right\|_{p_{i}, q_{i} ; v}, \quad i=0,1 .
$$

We use the Hölder inequality and the relation that $1 / r=(1-\theta) / r_{0}+\theta / r_{1}, w^{1 / r}=$ $w_{0}^{(1-\theta) / r_{0}} w_{1}^{\theta / r_{1}}$ to obtain

$$
\begin{aligned}
& s\left(\lambda_{T f_{n}, w}(s)\right)^{1 / r} \\
= & s\left(\int_{\left\{x ;\left|T f_{n}(x)\right|>s\right\}} w(x) d \mu(x)\right)^{1 / r} \\
\leq & s\left(\int_{\left\{x ;\left|T f_{n}(x)\right|>s\right\}} w_{0}(x) d \mu(x)\right)^{(1-\theta) / r_{0}}\left(\int_{\left\{x ;\left|T f_{n}(x)\right|>s\right\}} w_{1}(x) d \mu(x)\right)^{\theta / r_{1}} \\
= & {\left[s\left(\lambda_{T f_{n}, w_{0}}(s)\right)^{1 / r_{0}}\right]^{1-\theta}\left[s\left(\lambda_{T f_{n}, w_{1}}(s)\right)^{1 / r_{1}}\right]^{\theta} . }
\end{aligned}
$$

From (3.3) and (3.4),

$$
s\left(\lambda_{T f_{n}, w}(s)\right)^{1 / r} \leq C\left\|f_{n}\right\|_{p_{0}, q_{0} ; v}^{1-\theta}\left\|f_{n}\right\|_{p_{1}, q_{1} ; v}^{\theta} .
$$

Thus we obtain

$$
\left\|T f_{n}\right\|_{r, \infty ; w} \leq C\left\|f_{n}\right\|_{p_{0}, q_{0} ; v}^{1-\theta}\left\|f_{n}\right\|_{p_{1}, q_{1} ; v}^{\theta} .
$$

As for the quantity $\left\|f_{n}\right\|_{p_{0}, q_{0} ; v}$, we have

$$
\begin{aligned}
\left\|f_{n}\right\|_{p_{0}, q_{0} ; v} & =\left(\frac{q_{0}}{p_{0}} \int_{0}^{\infty}\left[t^{1 / p_{0}}\left(f_{n}\right)_{v}^{*}(t)\right]^{q_{0}} \frac{d t}{t}\right)^{1 / q_{0}} \\
& \leq\left(\frac{q_{0}}{p_{0}} \int_{0}^{v\left(E_{n}\right)}\left[t^{1 / p_{0}} f_{v}^{*}\left(2^{n}\right)\right]^{q_{0}} \frac{d t}{t}\right)^{1 / q_{0}} \\
& =f_{v}^{*}\left(2^{n}\right)\left(v\left(E_{n}\right)\right)^{1 / p_{0}} .
\end{aligned}
$$

Similarly as for the quantity $\left\|f_{n}\right\|_{p_{1}, q_{1} ; v}$,

$$
\left\|f_{n}\right\|_{p_{1}, q_{1} ; v} \leq f_{v}^{*}\left(2^{n}\right)\left(v\left(E_{n}\right)\right)^{1 / p_{1}} .
$$


By using the relation that $1 / p=(1-\theta) / p_{0}+\theta / p_{1}$, we obtain from (3.5),

$$
\left\|T f_{n}\right\|_{r, \infty ; w} \leq C f_{v}^{*}\left(2^{n}\right)\left(v\left(E_{n}\right)\right)^{1 / p} \leq C f_{v}^{*}\left(2^{n}\right) \cdot\left(2 \cdot 2^{n}\right)^{1 / p} .
$$

Consequently, from (3.2), (3.6) and Lemma 2.7 we obtain

$$
\|T f\|_{r, \infty ; w} \leq C \sum_{n=-\infty}^{\infty} f_{v}^{*}\left(2^{n}\right) \cdot 2^{n / p} \leq C\|f\|_{p, 1 ; v} .
$$

\section{ACKNOWLEDGEMENTS}

The authors would like to express their sincere gratitude to the referee for numerous suggestions to the original version of this paper.

\section{REFERENCES}

1. C. Bennett and R. Sharpley, Interpolation of operators, Academic Press, 1988. MR0928802 (89e:46001)

2. A. P. Calderón, Spaces between $L^{1}$ and $L^{\infty}$ and the theorem of Marcinkiewicz, Studia Math. 26 (1966), 273-299. MR0203444 (34:3295)

3. E. V. Ferreyra, On a negative result concerning interpolation with change of measures for Lorentz spaces, Proc. Amer. Math. Soc. 125 (1997), 1413-1417. MR1363418(97i:46055)

4. R. A. Hunt, On $L(p, q)$ spaces, Enseign. Math. 12 (1966), 249-276. MR0223874 (36:6921)

5. M. Kubo (Niwa) and S. Moritoh, An approach to Marcinkiewicz type interpolation theorem on weighted Lorentz spaces, Annual Report of Graduate School of Human Culture, Nara Women's Univ. 16 (2000), 259-265.

6. T. Sobukawa, Almost pointwise estimate and extrapolation theorem, Sci. Math. Jpn. 61 (2005), 153-158. MR2111550 (2005i:46022)

7. E. M. Stein and G. Weiss, Interpolation of operators with change of measures, Trans. Amer. Math. Soc. 87 (1958), 159-172. MR0092943 (19:1184d)

Department of Mathematics, Nara Women's University, Kitauoya-Nishimachi Nara, JAPAN 630-8506

E-mail address: moritoh@cc.nara-wu.ac.jp

Graduate School of Human Culture, Nara Women's University, Kitauoya-Nishimachi NARA, JAPAN 630-8506

E-mail address: yam.kubo@cc.nara-wu.ac.jp

Department of Education, Okayama University, 3-1-1 Tsushima-Naka Okayama, Japan $700-8530$

E-mail address: sobu@cc.okayama-u.ac.jp 